\title{
Solution of linear time-varying multi-delay systems via variational iteration method
}

\author{
Seyed Mehdi Mirhosseini-Alizamini, ${ }^{a, *}$, Sohrab Effatib,c ${ }^{\mathrm{b}}$ Aghileh Heydaria \\ a Department of Mathematics, Payame Noor University (PNU), P. O. Box 19395-3697, Tehran, Iran. \\ ${ }^{b}$ Center of Excellence on Soft Computing and Intelligent Information Processing (SCIIP), Ferdowsi University of Mashhad, \\ Iran. \\ ${ }^{c}$ Department of Applied Mathematics, Faculty of Mathematical Sciences, Ferdowsi University of Mashhad, Iran.
}

\begin{abstract}
This work presents an approximate solution method for the linear time-varying multi-delay systems and time delay logistic equation using variational iteration method. The method is based on the use of Lagrange multiplier for identification of optimal value of a parameter in a functional. This procedure is a powerful tool for solving large amount of problems. Also, it provides a sequence which converges to the solution of the problem without discretization of the variables. In this study, an idea is proposed that accelerates the convergence of the sequence which results from the variational iteration formula for solving systems of delay differential equations. Illustrative examples are included to demonstrate the validity and applicability of the technique. (c)2016 All rights reserved.
\end{abstract}

Keywords: Delay system, time varying, logistic equation, variational iteration method. 2010 MSC: 93C05, 30C70.

\section{Introduction}

Analysis, identification and optimal control of systems with time-delay has been a considerable concern. Time delay systems arise from an inherent time delay in the components of the system or

\footnotetext{
${ }^{*}$ Corresponding author

Email addresses: seyedmehdi_mirhosseini@yahoo.com (Seyed Mehdi Mirhosseini-Alizamini), s-effati@um.ac.ir.com (Sohrab Effati), a_heidari@pnu.ac.ir (Aghileh Heydari)
} 
a deliberate introduction of time delay into the system for control purposes. Delays occur frequently in biological, chemical, transportation, electronic, communication, manufacturing, traffic models, epidemiology, neuroscience, power systems, and control systems. Therefore, time-delay and multidelay systems are very important classes of systems whose control and optimization have been of interest to many investigators [14].

Many different methods have recently been introduced to solve time-delay systems, for example, orthogonal functions have received considerable attention in dealing with various problems of dynamic systems. Much progress has been made towards the solution of delay systems by using orthogonal functions. The approach is to convert the delay-differential equation to an algebraic through the use of operational matrices of integration and delay. In general, the computation of the delay systems via orthogonal functions is not in good agreement with the exact response of the system [19]. Special attention has been given to such applications as Walsh functions [3], hybrid functions [4] and Triangular functions [11. Special attention has been given to applications of wavelets [6], Adomian decomposition method (ADM) [2], homotopy perturbation method (HPM) [15], recurrent neural networks (RNN) [33] and others.

For these situations, variational iteration method (VIM), will be more effective. The VIM is strongly and simply capable of solving a large class of linear or nonlinear delay differential equations without the tangible restriction of sensitivity to the degree of the nonlinear term and it reduces the size of calculations as well. Its interactions are direct and straightforward.

On the other hand, in the context of numerical analysis, the VIM, which was proposed originally by He [8 10], has been proved by many authors to be a powerful mathematical tool for various kinds of linear and nonlinear ODEs or PDEs. In particular, He [11] demonstrated an application of VIM to a first order delay differential equation (DDE) modeling a population growth model. Other researches have also demonstrated the power of this method. There are some papers in the class of DDEs [1, 5, 7, 20, 22, 25. Unlike the traditional numerical methods, VIM needs no discretization, linearization, transformation, or perturbation. The VIM has been applied in a wide range of problems successfully, such as partial differential equations [22], fractional differential equations [23], integrodifferential equations [31] and nonlinear problems [26, 28. The main aim in this study is to effectively employ VIM to establish exact solutions and numerical results of linear and non-linear time-varying multi-delay systems and study the convergence of the method. To do so, we assume the delay term as restricted variations in VIM. This assumption is made in an effort to make it easier to find the Lagrange multipliers necessary for identifying the correction functional; imposing this assumption has noticeable effects on the ease with which VIM can be used for solving such equations. Furthermore, in this paper, we provide a derivation for an iteration form from a logistic nonlinear time-delay differential equation [29] model in which the rate of change of the population depends on three components: growth, death, and intra specific competition with the delay in the growth component. In our formulation, we incorporate the delay in the growth term in a manner consistent with the rate of instantaneous decline in the population given by the model. Some illustrative examples are given to demonstrate the accuracy and efficiency of the proposed method.

The paper is organized as follows. Section 2 is devoted to the formulation of linear time-varying multi-delay systems. A brief description of the VIM is given in Section 3 . The sufficient conditions are presented to guarantee the convergence of VIM, in this section. We review the main results concerning the dynamics of the classical logistic ODE and DDE equations and describe the VIM related model is Section 4. In Section 5, the numerical examples are simulated to show the reasonableness of our theory and demonstrate the performance of our network. Finally, we end this paper with conclusions in Section 6 . 


\section{Problem statement}

Consider the following linear time-varying multi-delay system:

$$
\begin{gathered}
\dot{x}(t)=E(t) x(t)+\sum_{j=1}^{r} F_{j}(t) x\left(t-\tau_{j}\right)+G(t) u(t), \quad t_{0} \leqslant t \leqslant t_{f}, \\
x\left(t_{0}\right)=x_{0}, \\
x(t)=\phi(t), \quad t_{0}-\tau_{j} \leqslant t<t_{0},
\end{gathered}
$$

where $x(t) \in \mathbb{R}^{n}, u(t) \in \mathbb{R}^{m}$, and $x_{0}$ is a constant specified vector at $t_{0}$. Moreover, $E(t), F_{j}(t), j=$ $1, \ldots, r$ and $G(t)$ are matrices of appropriate dimensions, and $\phi(t) \in C\left(\left[t_{0}-\tau_{j}, t_{0}\right], \mathbb{R}^{n}\right)$ is the continuous initial state function. The problem is to find $x(t), t_{0} \leqslant t \leqslant t_{f}$, satisfying Eqs. (2.1)-(2.3).

Delay differential Eqs.2.1)-2.3) are often solved using numerical methods, asymptotic solutions and graphical tools. In the next section we propose another analytic approximate method based on VIM for this purpose.

\section{Variational iteration method}

In this method, the problems are initially approximated with possible unknowns. To illustrate the basic concepts of the VIM we consider the following general equation with a multi-delay term:

$$
\mathcal{L}[x(t)]+\mathcal{N}\left[x(t), x\left(t-\tau_{1}\right), \ldots, x\left(t-\tau_{r}\right)\right]=g(t),
$$

where $\mathcal{L}$ is linear operator and $\mathcal{N}$ is nonlinear operator, $\tau_{j}, j=1, \ldots, r$ is the delay term, and $g(t)$ is an nonhomogeneous term.

The general Lagrange multiplier method was proposed by Inokuti et al. [13]. He [10] modified the general Lagrange multiplier method to an iteration method using a correction functional which can be written as follows:

$$
x_{n+1}(t)=x_{n}(t)+\int_{t_{0}}^{t} \Lambda(s)\left[\mathcal{L}\left[x_{n}(s)\right]+\mathcal{N}\left[\tilde{x}_{n}(s), \tilde{x}_{n}\left(s-\tau_{1}\right), \ldots, \tilde{x}_{n}\left(s-\tau_{r}\right)\right]-g(s)\right] d s,
$$

where $\Lambda$ is a general Lagrange multiplier, which can be identified optimally via variational theory, the subscript $n$ indexes the order of approximation and $\tilde{x}_{n}$ is considered as restricted variations [16], that is, $\delta \tilde{x}_{n}(t)=0, \delta \tilde{x}_{n}\left(t-\tau_{j}\right)=0, j=1, \ldots, r$.

With this assumption, the Lagrange multipliers can be easily and precisely obtained for linear problems. However, for nonlinear problems, they are not easy to obtain. In VIM, nonlinear terms $\tilde{x}_{n}$ are considered as restricted variations, a notion drawn from variational theory that allows the Lagrange multiplier can be more readily determined.

Since this method avoids discretization [8] of the problem, it is possible to find a closed from solution without any round-off error. The use of symbolic computation is necessary for finding the iterations. Therefore, we can successively approximate or even reach the exact solution by using

$$
x(t)=\lim _{n \rightarrow \infty} x_{n}(t) .
$$

In what follows, we apply VIM to some examples of linear and nonlinear multi-delay differential equations to illustrate the strength of the method and to establish exact solutions for these problems. 
For solving problem (2.1) by means of the VIM, the matrix $E=\left(e_{i j}\right)$ is decomposed into two matrices $D$ and $B$ such that $E=D+B$, where $D=\operatorname{diag}\left(e_{11}, e_{22}, \cdots, e_{n n}\right)$ and $B=E-D$. Let us define the operators $\mathcal{L}$ and $\mathcal{N}$ as follows:

$$
\begin{aligned}
\mathcal{L}[x(t)] & =\dot{x}(t)-D(t) x(t), \\
\mathcal{N}\left[\tilde{x}(t), \tilde{x}\left(t-\tau_{1}\right), \ldots, \tilde{x}\left(t-\tau_{r}\right)\right] & =-B(t) \tilde{x}(t)-\sum_{j=1}^{r} F_{j}(t) \tilde{x}\left(t-\tau_{j}\right) .
\end{aligned}
$$

Then we construct the following correction functional for $x$

$$
x_{n+1}(t)=x_{n}(t)+\int_{t_{0}}^{t} \Lambda(s)\left[\dot{x}_{n}(s)-D(s) x_{n}(s)-B(s) \tilde{x}_{n}(s)-\sum_{j=1}^{r} F_{j}(s) \tilde{x}_{n}\left(s-\tau_{j}\right)-g(s)\right] d s
$$

where $g(s)=G(s) u(s), \Lambda=\operatorname{diag}\left(\lambda_{1}, \lambda_{2}, \cdots, \lambda_{n}\right)$, in which $\lambda_{i}, i=1,2, \cdots, n$ are the Lagrange multipliers and $\tilde{x}_{n}$ denotes the restrictive variation, that is, $\delta \tilde{x}_{n}=0$. Note that although $B(s) \tilde{x}_{n}(s)$ is not a nonlinear term, we consider it as a nonlinear term.

By taking variation respect to $x_{n}$ and considering the restricted variation, we have

$$
\delta x_{n+1}(t)=\delta x_{n}(t)+\left.\Lambda(s) \delta x_{n}(s)\right|_{s=t}-\int_{t_{0}}^{t}(\dot{\Lambda}(s)+D(s) \Lambda(s)) \delta x_{n}(s) d s=0,
$$

the stationary conditions would be as follows

$$
\begin{aligned}
I+\left.\Lambda(s)\right|_{s=t} & =0, \\
\dot{\Lambda}(s)+D(s) \Lambda(s) & =0 .
\end{aligned}
$$

Here, the prime stands for differentiation with respect to the $s$. The latter equations can be written as follows:

$$
\begin{aligned}
1+\left.\lambda_{i}(s)\right|_{s=t} & =0, \\
\dot{\lambda}_{i}(s)+e_{i i}(s) \lambda_{i}(s) & =0 .
\end{aligned}
$$

For a fixed $i=1,2, \cdots, n$ we consider two cases. It can be readily identified that

$$
\Lambda(s)=-\exp \left\{\int_{t_{0}}^{t} e_{i i}(\xi) d \xi-\int_{t_{0}}^{s} e_{i i}(\xi) d \xi\right\}=-\exp \left\{\int_{s}^{t} e_{i i}(\xi) d \xi\right\}
$$

Hence we have

$$
\begin{aligned}
\Lambda(s) & =\operatorname{diag}\left(-\exp \left\{\int_{s}^{t} e_{11}(\xi) d \xi\right\}, \ldots,-\exp \left\{\int_{s}^{t} e_{n n}(\xi) d \xi\right\}\right) \\
& =-\exp \left\{\int_{s}^{t} \operatorname{diag}\left(e_{11}(\xi), \ldots, e_{n n}(\xi)\right) d \xi\right\} \\
& =-\exp \left\{\int_{s}^{t} D(\xi) d \xi\right\} .
\end{aligned}
$$

Then, the Lagrange multiplier can be obtain in the form

$$
\Lambda(s)=-\exp \left\{\int_{s}^{t} D(\xi) d \xi\right\}
$$


substituting the identified Lagrange multiplier, we obtain the following variational iteration formulation.

$$
x_{n+1}(t)=x_{n}(t)-\int_{t_{0}}^{t} \exp \left\{\int_{s}^{t} D(\xi) d \xi\right\}\left[\dot{x}_{n}(s)-E(s) x_{n}(s)-\sum_{j=1}^{r} F_{j}(s) x_{n}\left(s-\tau_{j}\right)-g(s)\right] d s
$$

with $x_{0}$ and $x(t)=\phi(t)$ for $t \in\left[t_{0}-\tau_{j}, t_{0}\right), j=1, \ldots, r$.

Beginning with an initial approximation $x_{0}(t)$, we obtain the successive approximations, and the exact solution can be obtained using

$$
x(t)=\lim _{n \rightarrow \infty} x_{n}(t) .
$$

Now, we show that the sequence $\left\{x_{n}(t)\right\}_{n=1}^{\infty}$ defined by VIM with $x_{0}(t)$ convergence to the solution of 2.1. To do this it was stated in the following theorem.

The sufficient conditions are presented to guarantee the convergence of VIM when applied to solve time-varying multi-delay, where the main point is that we prove the convergence of the recurrent sequence, which is generated by using VIM.

Theorem 3.1. Suppose that $E(t), F_{j}(t)(j=1, \ldots, r)$ and $g(t)$ belong to $C\left[t_{0}, t_{f}\right]$, then sequence $\left\{x_{n}(t)\right\}$ is convergent for $t \in\left[t_{0}, t_{f}\right]$.

Proof. Consider $\left\{x_{n}(t)\right\}$ as a sequence of (3.9), therefore, the exact solution $x(t)$ of 3.9 verifies

$$
x(t)=x(t)-\int_{t_{0}}^{t} \exp \left\{\int_{s}^{t} D(\xi) d \xi\right\}\left[\dot{x}(s)-E(s) x(s)-\sum_{j=1}^{r} F_{j}(s) x\left(s-\tau_{j}\right)-g(s)\right] d s .
$$

Introduce the error function

$$
e_{n+1}(t)=x_{n+1}(t)-x(t)
$$

Hence from (3.9)-(3.11) it follows that

$$
e_{n+1}(t)=e_{n}(t)-\int_{t_{0}}^{t} \exp \left\{\int_{s}^{t} D(\xi) d \xi\right\}\left[\dot{e}_{n}(s)-E(s) e_{n}(s)-\sum_{j=1}^{r} F_{j}(s) e_{n}\left(s-\tau_{j}\right)\right] d s,
$$

and

$$
\begin{aligned}
e_{n+1}(t)= & e_{n}(t)-\int_{t_{0}}^{t} \exp \left\{\int_{s}^{t} D(\xi) d \xi\right\} \dot{e}_{n}(s) d s \\
& +\int_{t_{0}}^{t} \exp \left\{\int_{s}^{t} D(\xi) d \xi\right\}\left[E(s) e_{n}(s)+\sum_{j=1}^{r} F_{j}(s) e_{n}\left(s-\tau_{j}\right)\right] d s
\end{aligned}
$$

By using part integration and $E=D+B$, we get

$$
\begin{aligned}
e_{n+1}(t)= & e_{n}(t)-\left[\left.\exp \left\{\int_{s}^{t} D(\xi) d \xi\right\} e_{n}(s)\right|_{s=t_{0}} ^{s=t}+\int_{t_{0}}^{t} \exp \left\{\int_{s}^{t} D(\xi) d \xi\right\} D(s) e_{n}(s) d s\right] \\
& +\int_{t_{0}}^{t} \exp \left\{\int_{s}^{t} D(\xi) d \xi\right\}[D(s)+B(s)] e_{n}(s) d s
\end{aligned}
$$




$$
+\int_{t_{0}}^{t} \exp \left\{\int_{s}^{t} D(\xi) d \xi\right\} \sum_{j=1}^{r} F_{j}(s) e_{n}\left(s-\tau_{j}\right) d s .
$$

Now, as the initialization of VIM is $x_{0}(t)=x\left(t_{0}\right)$, thus $x_{n}\left(t_{0}\right)=x\left(t_{0}\right)$, that is, $e_{n}\left(t_{0}\right)=0$, therefore

$$
\begin{aligned}
e_{n+1}(t)= & \int_{t_{0}}^{t} \exp \left\{\int_{s}^{t} D(\xi) d \xi\right\} B(s) e_{n}(s) d s \\
& +\int_{t_{0}}^{t} \exp \left\{\int_{s}^{t} D(\xi) d \xi\right\} \sum_{j=1}^{r} F_{j}(s) e_{n}\left(s-\tau_{j}\right) d s,
\end{aligned}
$$

and

$$
\begin{aligned}
\left\|e_{n+1}\right\| \leq & \|B\|\left\|\int_{t_{0}}^{t} \exp \left\{\int_{s}^{t} D(\xi) d \xi\right\}\right\|\left\|e_{n}(s)\right\| d s \\
& +\int_{t_{0}}^{t}\left\|\exp \left\{\int_{s}^{t} D(\xi) d \xi\right\}\right\| \sum_{j=1}^{r}\left\|F_{j}\right\|\left\|e_{n}\left(s-\tau_{j}\right)\right\| d s .
\end{aligned}
$$

Let $\sup \|D\|=L$, for $t_{0} \leq s \leq r \leq t_{f}$, we have

$$
\begin{aligned}
\left\|\exp \left\{\int_{s}^{t} D(\xi) d \xi\right\}\right\| & \leqslant \exp \left\|\left\{\int_{s}^{t} D(\xi) d \xi\right\}\right\| \\
& \leqslant \exp \left\{\int_{s}^{t}\|D(\xi)\| d \xi\right\} \\
& \leqslant \exp \left\{\int_{s}^{t} L d \xi\right\} \leqslant \exp \left(t_{f} L\right),
\end{aligned}
$$

hence, from (3.13) and (3.14), we obtain

$$
\left\|e_{n+1}(t)\right\| \leqslant M \int_{t_{0}}^{t}\left\|e_{n}(s)\right\| d s
$$

where $M=\exp \left(t_{f} L\right)\left(\|B\|+\sum_{j=1}^{r}\left\|F_{j}\right\|\right)$. Therefore

$$
\begin{aligned}
& \left\|e_{1}(t)\right\| \leqslant M \int_{t_{0}}^{t}\left\|e_{0}(s)\right\| d s \leqslant M \max \left\|e_{0}\left(s_{0}\right)\right\| \int_{t_{0}}^{t} d s \leqslant M \max \left\|e_{0}\left(s_{0}\right)\right\|\left(t-t_{0}\right), \\
& \left\|e_{2}(t)\right\| \leqslant M \int_{t_{0}}^{t}\left\|e_{1}(s)\right\| d s \leqslant M^{2} \max \left\|e_{0}\left(s_{0}\right)\right\| \int_{t_{0}}^{t}\left(s-t_{0}\right) d s \leqslant M^{2} \max \left\|e_{0}\left(s_{0}\right)\right\| \frac{\left(t-t_{0}\right)^{2}}{2 !}, \\
& \left\|e_{3}(t)\right\| \leqslant M \int_{t_{0}}^{t}\left\|e_{2}(s)\right\| d s \leqslant M^{3} \max \left\|e_{0}\left(s_{0}\right)\right\| \int_{t_{0}}^{t} \frac{\left(s-t_{0}\right)^{2}}{2 !} d s \leqslant M^{3} \max \left\|e_{0}\left(s_{0}\right)\right\| \frac{\left(t-t_{0}\right)^{3}}{3 !} .
\end{aligned}
$$

From this development, it follows that

$$
\left\|e_{n}(t)\right\| \leqslant \max \left\|e_{0}\left(s_{0}\right)\right\| \frac{\left(M\left(t-t_{0}\right)\right)^{n}}{n !} \leqslant \max \left\|e_{0}\left(s_{0}\right)\right\| \frac{(M h)^{n}}{n !}, \quad s_{0} \in\left[t_{0}, t_{f}\right]
$$

where $h=t_{f}-t_{0}$. As $\lim _{n \rightarrow \infty} \frac{h^{n}}{n !}=0$ with fixed $h>0$, it follows that, as $n \rightarrow \infty,\left\|e_{n}(t)\right\| \rightarrow 0$, that is, the sequence $\left\{x_{n}\right\}$ convergence to the exact solution $x(t)$. The proof is complete. 


\section{Application of the VIM for nonlinear time-delay system}

In this section, we shall introduce a new reliable way for choosing the initial approximations in VIM to solve non-linear time-delay system in biology, which is reduced to a recurrent sequence.

The delay logistic equations have been extensively used as models in biology an other sciences, with particular emphasis on population dynamics. The logistic equation of population growth was proposed in the first half of the 19th century by the Belgian mathematician Pierre-Francois Verhulst (1838) [29] as a potential solution to the dilemma of Malthusian exponential growth:

$$
\dot{x}(t)=r x(t)\left(1-\frac{x(t)}{k}\right),
$$

where $r>0$ is the intrinsic growth rate and $k>0$ is the carrying capacity of the population.

Logistic equation (4.1) only assumes that the growth rate of a population at any time $t$ depends on the relative number of individuals at that time. In practice, the process of reproduction is not instantaneous. Hutchinson [12 assumed egg formation to occur $\tau$ units of time before hatching and proposed the following delayed logistic equation

$$
\dot{x}(t)=r x(t)\left(1-\frac{x(t-\tau)}{k}\right),
$$

subject to the initial condition:

$$
x(t)=\alpha, \quad-\tau \leqslant t \leqslant 0,
$$

where $r$ and $k$ have the same biological meaning as $(4.1)$ and $\tau>0$ is the time delay. Here Eqs. (4.2)- 4.3) is a special case of Eqs. (2.1)- 2.3), such that $u(t)=0$.

When $\tau=0$, the solution can be expressed in a closed form:

$$
x(t)=\frac{k}{1+\left(\frac{k}{\alpha}-1\right) \exp (-r t)},
$$

which tends to $k$ as $t \rightarrow+\infty$, it holds $x(t-\tau)=\alpha$, and then the original becomes:

$$
\dot{x}(t)=r x(t)\left(1-\frac{\alpha}{k}\right), \quad x(0)=\alpha,
$$

which has the solution:

$$
x(t)=\alpha \exp \left[-r\left(\frac{\alpha}{k}-1\right) t\right]
$$

with the property:

$$
\lim _{t \rightarrow+\infty} x(t) \rightarrow\left\{\begin{array}{lll}
0, & \text { when } & \alpha>k>0, \\
\alpha, & \text { when } & \alpha=k, \\
+\infty, & \text { when } & 0<\alpha<k .
\end{array}\right.
$$

Using the properties of Eq. 4.2, the initial condition was proposed as follows [16]:

$$
x(0) \rightarrow\left\{\begin{array}{l}
\alpha, \text { when }-\tau \leqslant t \leqslant 0, \\
x_{\infty}+\left(\alpha-x_{\infty}\right) \exp (-\beta t), \text { when } t \leqslant 0 .
\end{array}\right.
$$


Delayed biological systems have received much attention from biologists and mathematicians. The applications of delay equations to biomodeling in many cases is associated which studies of dynamic phenomena like oscillations, bifurcation and chaotic behavior. Time delay represents an additional level of complexity that can be incorporated in a more detailed analysis of particular system. Properties of Eq. (4.2) were studied very extensively by various authors [12, 16].

Now, according to the VIM (3.6), its correction functional of Eq. (4.2) can be written in the form

$$
x_{n+1}(t)=x_{n}(t)+\int_{0}^{t} \Lambda(s)\left[\dot{x}_{n}(s)-r x_{n}(s)+\frac{r}{k} \tilde{x}_{n}(s) \tilde{x}_{n}(s-\tau)\right] d s .
$$

According to the Eq. (3.8), the Lagrange multiplier, therefore, can be obtained in the form

$$
\Lambda(s)=-\exp (r(t-s))
$$

substituting the identified Lagrange multiplier into 4.5), we obtain the following variational iteration formula

$$
x_{n+1}(t)=x_{n}(t)-\int_{0}^{t} \exp (r(t-s))\left[\dot{x}_{n}(s)-r x_{n}(s)+\frac{r}{k} \tilde{x}_{n}(s) \tilde{x}_{n}(s-\tau)\right] d s .
$$

Therefore, starting with $x(0)$, the approximation solution is given as $x(t)=\lim _{n \rightarrow \infty} x_{n}(t)$. The convergence of this system is similar to convergence of the VIM discussed in Section 3 .

\section{Illustrative examples}

The following various examples are given to illustrate the simplicity and efficiency of the proposed method. The codes are developed using symbolic computation software MATLAB and the calculations are implemented on a machine with Intel core 2 Due processor 2.50 Ghz and 4 GB RAM.

Example 5.1. Consider the following multi-delay system:

$$
\begin{gathered}
\dot{x}(t)=t x(t-0.4)+x(t-0.8)+1, \quad 0 \leqslant t \leqslant 1, \\
x(t)=0, \quad t \leqslant 0 .
\end{gathered}
$$

The exact solution of this example is [18]

$$
x(t)=\left\{\begin{array}{l}
t, \quad 0 \leqslant t<0.4, \\
\frac{2}{5}+(t-0.4)+\frac{1}{5}(t-0.4)^{2}+\frac{1}{3}(t-0.4)^{3}, \quad 0.4 \leqslant t<0.8, \\
\frac{64}{75}+\frac{33}{25}(t-0.8)+\frac{11}{10}(t-0.8)^{2}+\frac{29}{75}(t-0.8)^{3}+\frac{7}{60}(t-0.8)^{4}+\frac{1}{15}(t-0.8)^{5}, \quad 0.8 \leqslant t \leqslant 1 .
\end{array}\right.
$$

To solve Eq. (5.1) and (5.2) by means of VIM, we can obtain the Lagrange multiplier by using Eq. (3.8), $\lambda(s)=-1$.

Now, the following variational iteration formula can be obtained

$$
x_{n+1}(t)=x_{n}(t)-\int_{0}^{t}\left[\dot{x}_{n}(s)-s x_{n}(s-0.4)-x_{n}(s-0.8)-1\right] d s .
$$


We start with an initial approximation $x_{0}(t)=0$, and by using the above iteration formula (5.3), we can obtain directly the components of the solution $x(t)$. This in turn yields the components:

$$
\begin{aligned}
x_{1}(t) & =0-\int_{0}^{t}(-1) d s=t, \quad 0 \leqslant t<0.4, \\
x_{1}(t) & =t-\int_{0.4}^{t}[1-s(s-0.4)-1] d s \\
& =\frac{2}{5}+(t-0.4)+\frac{1}{5}(t-0.4)^{2}+\frac{1}{3}(t-0.4)^{3}, \quad 0.4 \leqslant t<0.8 .
\end{aligned}
$$

Similarly

$$
\begin{aligned}
x_{1}(t)= & \frac{64}{75}+\frac{33}{25}(t-0.8)+\frac{11}{10}(t-0.8)^{2}+\frac{29}{75}(t-0.8)^{3}+\frac{7}{60}(t-0.8)^{4} \\
& +\frac{1}{15}(t-0.8)^{5}, \quad 0.8 \leqslant t \leqslant 1 .
\end{aligned}
$$

Consequently, the exact solution may be obtained by using one order iteration, that is, $x(t)=x_{1}(t)$. Also, the exact solution for the delay system obtained by the presented method is compared with other methods, since hybrid of block pulse functions and Taylor series $\left(b_{n m}(t), n=1, \cdots, N, m=\right.$ $0,1, \cdots, M-1)$ [18, where $n$ and $m$ are the order of block pulse functions and Taylor polynomials, respectively. Here the solution of this example is obtained with hybrid functions by choosing $N=3$ and $M=6$. It to be noted that exact solutions obtained in the example cannot be obtained either with orthogonal functions (see, e.g., [3]). In general, the computation of the delay systems via orthogonal functions is not in good agreement with the exact response of the system.

Example 5.2. Consider the following delay system with delay in both control and state:

$$
\begin{gathered}
\dot{x}(t)=-x(t)-2 x\left(t-\frac{1}{4}\right)+2 u\left(t-\frac{1}{4}\right), \quad 0 \leqslant t \leqslant 1, \\
x(t)=u(t)=0, \quad-\frac{1}{4} \leqslant t \leqslant 0 . \\
u(t)=1, \quad t>0 .
\end{gathered}
$$

Although the above system has a delay in control, the method described here, can be used.

The exact solution is [4]

$$
x(t)=\left\{\begin{array}{l}
0, \quad 0 \leqslant t<\frac{1}{4}, \\
2-2 \exp \left(\frac{1}{4}-t\right), \quad \frac{1}{4} \leqslant t<\frac{1}{2}, \\
-2-2 \exp \left(\frac{1}{4}-t\right)+(2+4 t) \exp \left(\frac{1}{2}-t\right), \quad \frac{1}{2} \leqslant t<\frac{3}{4}, \\
6-2 \exp \left(\frac{1}{4}-t\right)+(2+4 t) \exp \left(\frac{1}{2}-t\right)-\left(\frac{17}{4}+2 t+4 t^{2}\right) \exp \left(\frac{3}{4}-t\right), \quad \frac{3}{4} \leqslant t \leqslant 1 .
\end{array}\right.
$$

The Lagrange multiplier can be readily identified as follows

$$
\lambda(s)=-\exp \left\{\int_{0}^{t}(-1) d \xi-\int_{0}^{s}(-1) d \xi\right\}=-\exp (s-t) .
$$


We obtain the iteration formula

$$
x_{n+1}(t)=x_{n}(t)-\int_{\frac{1}{4}}^{t} \exp (s-t)\left[\dot{x}_{n}(s)+x_{n}(s)+2 x_{n}\left(s-\frac{1}{4}\right)-2 u\left(s-\frac{1}{4}\right)\right] d s .
$$

Starting with $x_{0}(t)=0$ and using the iteration formula, we find

$$
\begin{aligned}
x_{1}(t) & =0, \quad 0 \leqslant t<\frac{1}{4} \\
x_{1}(t) & =0-\int_{\frac{1}{4}}^{t} \exp (s-t)[-2] d s=2-2 \exp \left(\frac{1}{4}-t\right), \quad \frac{1}{4} \leqslant t<\frac{1}{2}, \\
x_{1}(t) & =2-2 \exp \left(\frac{1}{4}-t\right)-\int_{\frac{1}{2}}^{t} \exp (s-t)\left[4-4 \exp \left(\frac{1}{2}-s\right)\right] d s \\
& =-2-2 \exp \left(\frac{1}{4}-t\right)+(2+4 t) \exp \left(\frac{1}{2}-t\right), \quad \frac{1}{2} \leqslant t<\frac{3}{4} .
\end{aligned}
$$

Similarly in $\frac{3}{4} \leqslant t \leqslant 1$, we obtained

$$
x_{1}(t)=6-2 \exp \left(\frac{1}{4}-t\right)+(2+4 t) \exp \left(\frac{1}{2}-t\right)-\left(\frac{17}{4}+2 t+4 t^{2}\right) \exp \left(\frac{3}{4}-t\right) .
$$

Therefore, the exact solution may be obtained by using $x(t)=x_{1}(t)$. In Table 1 a comparison is made between the exact solution and the approximate solution of $x(t)$ for one order iteration, that is, $n=1$. The approximate value of $x(t)$ on $[0,1]$ is the same as the exact solution. The optimal state $x(t)$ is depicted in Fig. 1. The results obtained via Lagrange polynomials [4, Triangular function [11] and adaptive Legendre-Gauss-Radau collocation method [17] are to that shown in Table 1. We mention in [17], $N$ is the number of subintervals of the adaptive collocation method. Here, the solution of this example is obtained by choosing $N=7$.

Table 1: Comparison of the VIM for Example 5.2, and estimated an exact value of $x(t)$.

\begin{tabular}{lccccc}
\hline $\mathrm{t}$ & LaP [4] & TFs [11] & LeGR [17] & Present method & Exact \\
\hline 0 & 0 & 0 & 0 & 0 & 0 \\
0.125 & 0 & 0 & 0 & 0 & 0 \\
0.25 & 0 & 0 & 0 & 0 & 0 \\
0.375 & 0.2348581 & 0.2350106 & 0.2350061 & 0.2350061 & 0.2350061 \\
0.5 & 0.4082723 & 0.4424063 & 0.4423984 & 0.4423984 & 0.4423984 \\
0.625 & 0.5485504 & 0.5966848 & 0.5966575 & 0.5966575 & 0.5966575 \\
0.75 & 0.6591615 & 0.6809826 & 0.6809425 & 0.6809425 & 0.6809425 \\
0.875 & 0.7433335 & 0.7120013 & 0.7119399 & 0.7119399 & 0.7119399 \\
1.0 & 0.8041378 & 0.7118111 & 0.7117428 & 0.7117428 & 0.7117428 \\
\hline
\end{tabular}




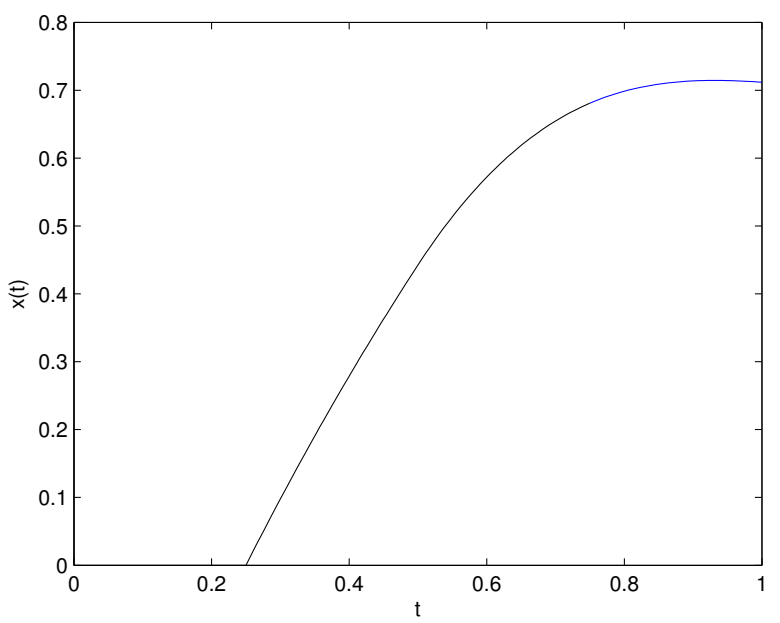

Figure 1: The state, when $n=1$ for Example 5.2

Example 5.3. Consider the time-varying multi-delay system described by

$$
\left(\begin{array}{c}
\dot{x}_{1}(t) \\
\dot{x}_{2}(t)
\end{array}\right)=\left(\begin{array}{cc}
t & 1 \\
t & 2 t
\end{array}\right)\left(\begin{array}{l}
x_{1}\left(t-\frac{1}{3}\right) \\
x_{2}\left(t-\frac{1}{3}\right)
\end{array}\right)+\left(\begin{array}{cc}
2 & t \\
t^{2} & 0
\end{array}\right)\left(\begin{array}{l}
x_{1}\left(t-\frac{2}{3}\right) \\
x_{2}\left(t-\frac{2}{3}\right)
\end{array}\right)+\left(\begin{array}{l}
0 \\
1
\end{array}\right) u(t)
$$

with

$$
x_{1}(t)=x_{2}(t)=u(t)=0, \quad-\frac{2}{3} \leqslant t \leqslant 0,
$$

and

$$
u(t)=2 t+1, \quad t>0 .
$$

The exact solutions are [4]

$$
x_{1}(t)=\left\{\begin{array}{l}
0, \quad 0 \leqslant t<\frac{1}{3} \\
\frac{7}{162}-\frac{2}{9} t+\frac{1}{6} t^{2}+\frac{1}{3} t^{3}, \quad \frac{1}{3} \leqslant t<\frac{2}{3}, \\
\frac{11}{162}-\frac{58}{243} t+\frac{31}{162} t^{2}+\frac{1}{9} t^{3}+\frac{7}{72} t^{4}+\frac{1}{6} t^{5}, \quad \frac{2}{3} \leqslant t \leqslant 1
\end{array}\right.
$$

and

$$
x_{2}(t)=\left\{\begin{array}{l}
t+t^{2}, \quad 0 \leqslant t<\frac{1}{3}, \\
\frac{5}{486}+t+\frac{7}{9} t^{2}+\frac{2}{9} t^{3}+\frac{1}{2} t^{4}, \quad \frac{1}{3} \leqslant t<\frac{2}{3}, \\
\frac{1}{486}+t+\frac{200}{243} t^{2}+\frac{20}{81} t^{3}+\frac{29}{72} t^{4}+\frac{1}{9} t^{5}+\frac{1}{6} t^{6}, \quad \frac{2}{3} \leqslant t \leqslant 1 .
\end{array}\right.
$$

The Lagrange multiplier can be readily identified $\lambda(s)=(-1,-1)$. The corresponding variational iteration formula reads

$$
x_{1 n+1}(t)=x_{1 n}(t)-\int_{0}^{t}\left[\dot{x}_{1 n}(s)-s x_{1 n}\left(s-\frac{1}{3}\right)-x_{2 n}\left(s-\frac{1}{3}\right)-2 x_{1 n}\left(s-\frac{2}{3}\right)-s x_{2 n}\left(s-\frac{2}{3}\right)\right] d s,
$$

and

$$
x_{2 n+1}(t)=x_{2 n}(t)-\int_{0}^{t}\left[\dot{x}_{2 n}(s)-s x_{1 n}\left(s-\frac{1}{3}\right)-2 s x_{2 n}\left(s-\frac{1}{3}\right)-s^{2} x_{1 n}\left(s-\frac{2}{3}\right)-u(s)\right] d s .
$$


Here, we apply the proposed method described in Section 3 , for obtaining $x_{1}(t)$ and $x_{2}(t)$. By choosing $x_{0}(t)=(0,0)$, the above iteration formula yields exact solutions. That is, $x(t)=\left(x_{1}(t), x_{2}(t)\right)^{T}$. Consequently, the exact solution may be obtained by using one order iteration. The optimal states $x_{1}(t)$ and $x_{2}(t)$ are depicted in Figs. 2 and 3 . Also, the exact solution for the delay system obtained by the presented method is compared with other methods since hybrid of block pulse functions and Taylor series $\left(b_{n m}(t), n=1, \cdots, N, m=0,1, \cdots, M-1\right)$ [18], where $n$ and $m$ are the order of block pulse functions and Taylor polynomials, respectively. Here is the solution of this example obtained with hybrid functions by choosing $N=3$ and $M=7$.

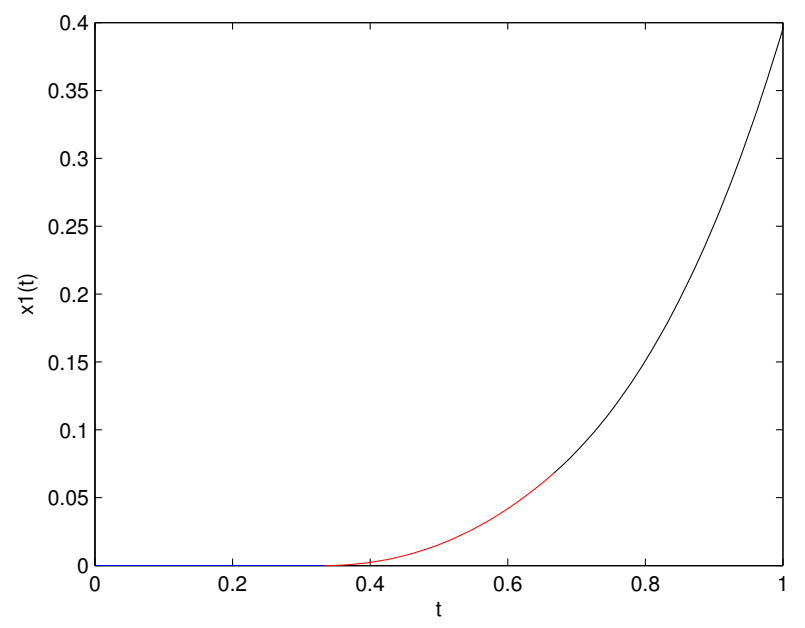

Figure 2: The state $x_{1}(t)$, when $n=1$ for Example 5.3

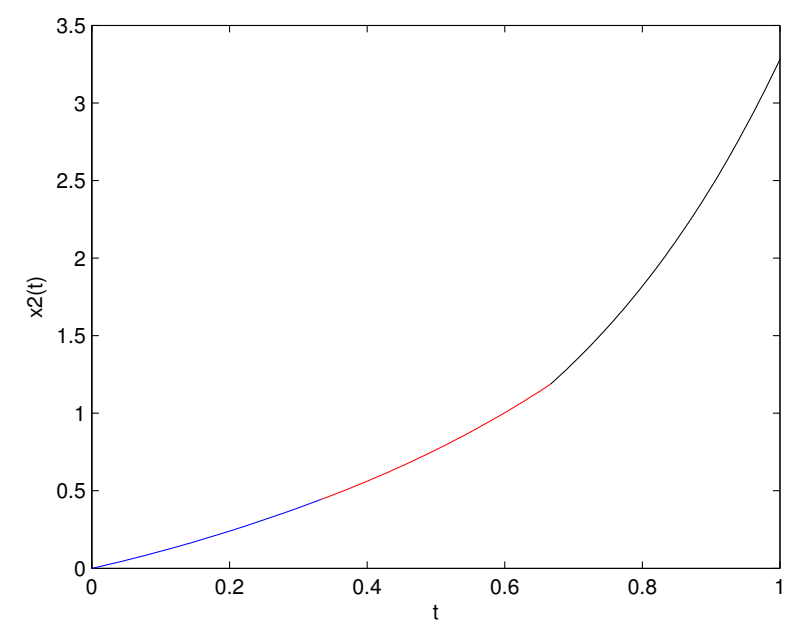

Figure 3: The state $x_{2}(t)$, when $n=1$ for Example 5.3

Example 5.4. Consider the delay system [30] described by

$$
\left(\begin{array}{l}
\dot{x}_{1}(t) \\
\dot{x}_{2}(t)
\end{array}\right)=\left(\begin{array}{cc}
t^{2}+1 & -t^{2} \\
0 & -9
\end{array}\right)\left(\begin{array}{l}
x_{1}(t) \\
x_{2}(t)
\end{array}\right)+\left(\begin{array}{cc}
1 & -1 \\
9 & 0
\end{array}\right)\left(\begin{array}{l}
x_{1}\left(t-\frac{1}{2}\right) \\
x_{2}\left(t-\frac{1}{2}\right)
\end{array}\right)+\left(\begin{array}{c}
4 t+3 \\
8 t+15
\end{array}\right) u(t),
$$


with

$$
x(t)=\left(\begin{array}{c}
t^{2}-1 \\
t^{2}+1
\end{array}\right), \quad-\frac{1}{2} \leqslant t \leqslant 0
$$

and

$$
u(t)=1, \quad t \geq 0 .
$$

According to the VIM, to solve this example, we can construct a correction functional as follows:

$$
\begin{aligned}
x_{1 n+1}(t)= & x_{1 n}(t)+\int_{0}^{t} \lambda_{1}(s)\left[\dot{x}_{1 n}(s)-\left(s^{2}+1\right) x_{1 n}(s)+s^{2} x_{2 n}(s)\right. \\
& \left.\left.-x_{1 n}\left(s-\frac{1}{2}\right)+x_{2 n}\left(s-\frac{1}{2}\right)-(4 s+3) u(s)\right]\right] d s
\end{aligned}
$$

and

$$
x_{2 n+1}(t)=x_{2 n}(t)+\int_{0}^{t} \lambda_{2}(s)\left[\dot{x}_{2 n}(s)+9 x_{2 n}(s)-9 x_{1 n}\left(s-\frac{1}{2}\right)-(8 s+15) u(s)\right] d s .
$$

Thus, using the stationary conditions lead to Lagrange multiplier $\lambda_{1}(s)=-\exp (t-s)$ and $\lambda_{2}(s)=$ $-\exp (9(s-t))$. Substitution of this value of the Lagrange multiplier into the functional (5.13) yields the iteration formula for $n \geq 0$.

Selection of $x_{10}(t)=t^{2}-1$ and $x_{20}(t)=t^{2}+1$ from the given initial condition and using the iteration formula (5.13) and (5.14) yields the successive approximations of $x(t)=\left(x_{1}(t), x_{2}(t)\right)^{T}$

$0 \leq t<\frac{1}{2}$ :

$$
\begin{aligned}
x_{11}(t) & =t^{2}-1-\int_{0}^{t} \exp (t-s)\left[-2 s+s^{4}\right] d s \\
& =21+22 t+13 t^{2}+4 t^{3}+t^{4}-22 \exp (t)
\end{aligned}
$$

and

$$
\begin{aligned}
x_{21}(t)= & t^{2}+1-\int_{0}^{t} \exp (9(s-t))\left[-\frac{1941}{16}-\frac{219}{2} s-\frac{135}{2} s^{2}-18 s^{3}-9 s^{4}+198 \exp \left(s-\frac{1}{2}\right)\right] d s \\
= & \frac{465383}{34992}+\frac{5167}{486} t+\frac{427}{54} t^{2}+\frac{14}{9} t^{3}+t^{4}+\frac{99}{5} \exp \left(-9 t-\frac{1}{2}\right) \\
& -\frac{99}{5} \exp \left(t-\frac{1}{2}\right)-\frac{430391}{34992} \exp (-9 t) .
\end{aligned}
$$

$\frac{1}{2} \leq t \leq 1:$

$$
\begin{aligned}
x_{12}(t)= & \frac{40283583}{34992}+\frac{20256383}{17946} t+\frac{20458343}{34992} t^{2}+\frac{92791}{486} t^{3}+\frac{2467}{54} t^{4} \\
& +\frac{68}{9} t^{5}+t^{6}+\frac{99}{50} \exp (-9 t+4)+\frac{99}{50}\left(t^{2}+\frac{1}{5} t+\frac{1}{50}\right) \exp \left(-9 t-\frac{1}{2}\right) \\
& +\frac{99}{5} \exp (t-1)+\frac{33}{5} t^{3} \exp \left(t-\frac{1}{2}\right)-\frac{430391}{34992} \exp \left(-9 t+\frac{9}{2}\right)-22 \exp (t)
\end{aligned}
$$




$$
\begin{aligned}
& +\frac{430391}{349920}\left(t^{2}+\frac{1}{5} t+\frac{1}{50}\right) \exp (-9 t)-\frac{79611513}{43740} \exp \left(t-\frac{1}{2}\right) \\
& -\frac{297}{25} \exp (t-1)-\frac{3663}{5000} \exp \left(t-\frac{11}{2}\right)+\frac{15924467}{34992000} \exp (t-5),
\end{aligned}
$$

and

$$
\begin{aligned}
x_{22}(t)= & \frac{465511}{34992}-\frac{5159}{486} t+\frac{431}{54} t^{2}+\frac{14}{9} t^{3}+t^{4}-\frac{99}{5} \exp \left(t-\frac{1}{2}\right) \\
& -\frac{430391}{34992} \exp (-9 t)+\left(\frac{539}{5}-176 t\right) \exp \left(-9 t-\frac{1}{2}\right)-\frac{61}{4374} \exp \left(\frac{9}{2}-9 t\right) .
\end{aligned}
$$

It can be checked that the VIM (one order iteration) solutions are equal to the exact solutions.

Example 5.5. In this example we consider the following non-linear delay differential equation which is a well-known equation from biology.

$$
\begin{aligned}
\dot{x}(t) & =4 x(t)(1-3 x(t-0.1)), \\
x(0) & =\frac{1}{2}+\frac{1}{2} e^{-2 t}, \quad t \geq 0, \\
x(t) & =1, \quad-0.3 \leqslant t<0 .
\end{aligned}
$$

According to Eq. 4.5, we obtain the iteration formula

$$
x_{n+1}(t)=x_{n}(t)-\int_{0}^{t} \exp (4(t-s))\left[\dot{x}_{n}(s)-4 x_{n}(s)+12 x_{n}(s) x_{n}(s-0.3)\right] d s .
$$

Starting with $x_{0}(t)$ and using the iteration formula, we find $x_{1}, x_{2}$ and $x_{3}$.

Table 2: The numerical results for example 5.5 using the VIM.

\begin{tabular}{lccc}
\hline $\mathrm{t}$ & VIM & VIM & dde \\
& $x$ (iteration 2) & $x$ (iteration 3) & $x$ \\
\hline 0 & 1 & 1 & 1 \\
0.5 & 0.58965802 & 0.60735112 & 0.60735110 \\
1 & 0.17082620 & 0.19054234 & 0.19054339 \\
1.5 & 0.19839652 & 0.19901523 & 0.19901825 \\
2 & 0.22546785 & 0.22681425 & 0.22680423 \\
2.5 & 0.23685240 & 0.23615016 & 0.23615016 \\
3 & 0.25386117 & 0.25360017 & 0.25360017 \\
3.5 & 0.25609911 & 0.25600903 & 0.25600903 \\
4 & 0.25746020 & 0.25700010 & 0.25700010 \\
4.5 & 0.25753215 & 0.25700032 & 0.25700032 \\
5 & 0.25763145 & 0.25700046 & 0.25700046 \\
\hline
\end{tabular}

In Table 2 a comparison is made between the numerical results obtained by Matlab toolbox for solving DDE [27] which used the Runge-Kutta triple BS $(2,3)$ method and the approximate solution of $x(t)$ for $n=2$ (iteration 2) and $n=3$ (iteration 3 ). The approximate value of $x(t)$ on $[2, \infty)$ is the same as the numerical results. 


\section{Conclusions}

This article deals with VIM for solving a linear and nonlinear time-varying multi-delay systems and nonlinear delay differential equation namely delay logistic equation. We described the method and used it in some test examples in order to show its applicability and validity in comparison with other methods and exact solutions. We achieved satisfactory approximations with a few number of iterations, which reveals the efficiency of the method. Moreover, since this method do not need the discretization of the variables, there is no computation round off errors and one is not faced with necessity of large computer memory and time.

\section{References}

[1] Z. Ayati, J. Biazar, S. Ebrahimi, A new homotopy perturbation method for solving two-dimensional reactiondiffusion brusselator system, J. Math. comput. sci., 15 (2015), 195-203. 1 .

[2] L. Blanco-Cocom, A. G. Estrella, E. Avila-Vales, Solving delay differential systems with history functions by the Adomian decomposition method, Appl. Math. Comput., 218 (2012), 5994-6011. 1

[3] W. L. Chen, Walsh series analysis of multi-delay systems, J. Franklin Institute, 313 (1982), $207-217$. 1. 5.1

[4] K. B. Datta, B. M. Mohan, Orthogonal functions in systems and control, World Scientific Publishing Co., Inc., River Edge, NJ, (1995). 1, 5.2, $1,5.3$

[5] Y. Dong, J. Liu, S. Mei, Observer design for a class of nonlinear discrete-time systems with time-delay, Kybernetika, 49 (2013), 341-358. 1

[6] M. Ghasemi, M. Tavassoli Kajani, Numerical solution of time-varying delay systems by Chebyshev wavelets, Appl. Math. Model., 35 (2011), 5235-5244. 1

[7] M. Gubes, H. Alpaslan Peker, G. Oturanc, Application of differential transform method for el nino southern oscillation (ENSO) model with compared Adomian decomposition and variational iteration methods, J. Math. Computer Sci., 15 (2015), 167-178. 1

[8] J. H. He, Variational iteration method for delay differential equations, Commun. Nonlinear Sci. Numer. Simul., 2 (1997), 235-236. 1, 3

[9] J. H. He, Some asymptotic methods for strongly nonlinear equations, Int. J. Mod. Phys. B, 20 (2006), 1141-1199.

[10] J. H. He, G. C. Wu, F. Austin, Variational iteration method which should be followed, Nonlinear Sci. Lett. A, 2010 (2010), 30 pages. 1], 3

[11] S. M. Hoseini, H. R. Marzban, Analysis of time-varying delay systems via triangular functions, Appl. Math. Comput., 217 (2011), 7432-7441. 1, 5.2, 1 ,

[12] G. E. Hutchinson, Circular causal system in ecology, Ann. N. Y. Acad. Sci., 50 (1948), 221-246. 4. 4

[13] M. Inokuti, H. Sekine, T. Mura, S. Nemat-Nasser, General use of the Lagrange multiplier in nonlinear mathematical physics, Variational Method in the Mechanics of Solids, edited by S. NEMAT-NASSER, Pergamon, (1980), 156-162. 3

[14] M. Jamshidi, C. M. Wang, A computational algorithm for large-scale nonlinear time-delays systems, IEEE Trans. Systems Man Cybernet., 14 (1984), 2-9. 1

[15] M. M. Khader, Introducing an efficient modification of the homotopy perturbation method by using Chebyshev polynomials, Arab J. Math. Sci., 18 (2012), 61-71. 1

[16] H. Khan, S. J. Liao, R. N. Mohapatra, K. Vajravelu, An analytical solution for a nonlinear time-delay model in biology, Commun. Nonlinear Sci. Numer. Simul., 14 (2009), 3141-3148. 3, 4, 4

[17] M. Maleki, I. Hshim, S. Abbasbandy, Solution of time-varying delay systems using an adaptive collocation method, Appl. Math. Comput., 219 (2012), 1434-1448. 5.2, 1

[18] H. R. Marzban, M. Razzaghi, Solution of multi-delay systems using hybrid of block-pulse functions and Taylor series, J. Sound Vibration, 292 (2006), 954-963. 5.1, 5.1, 5.3

[19] H. R. Marzban, M. Shahsiah, Solution of piecewise constant delay systems using hybrid of block-pulse and Chebyshev polynomials, Optimal Control Appl. Methods, 32 (2011), 647-659. 1

[20] M. Matinfar, M. Saeidy, A new analytical method for solving Hamilton-Jacobi-Bellman equation, J. Math. Comput. Sci., 11 (2014), 252-263. 1

[21] S. M. Mirhosseini-Alizamini, S. Effati, A. Heydari, An iterative method for suboptimal control of linear time-delayed systems, Systems Control Lett., 82 (2015), 40-50. 
[22] S. T. Mohyud-Din, A. Yildirim, Variational iteration method for delay differential equations using He's polynomials, Zeitschrift für Naturforschung, 65 (2010), 1045-1048. 1

[23] S. Momani, Z. M. Odibat, Analytical approach to linear fractional partial differential equations arising in fluid mechanics, Phys. Lett. A, 355 (2006), 271-279. 1

[24] Z. M. Odibat, A study on the convergence of variational iteration method, Math. Comput. Modelling, 51 (2010), 1181-1192.

[25] Y. M. Rangkuti, M. S. Noorani, The exact solution of delay differential equations using coupling variational iteration with Taylor series and small term, Bul. Math., 4 (2012), 1-15. 1

[26] H. Saberi nik, M. S. Zahedi, R. Buzhabadi, S. Effati, Homotopy perturbation method and He's polynomials for solving the porous media equation, Comput. Math. Model., 24 (2013), 279-292. 1

[27] L. F. Shampine, S. Thompson, Solving DDEs in MATLAB, Appl. Numer. Math, 37 (2001), $441-458$. 5.5

[28] M. Shirazian, S. Effati, Solving a class of nonlinear optimal control problems via He's variational iteration method, Int. J. Control Automat. Sys., 10 (2012), 249-256. 1

[29] P. F. Verhulst, Notice sur la loi lu population pursuit dans son accroissment, Coraesp. Math. Phys, 10 (1838), 113-121.1., 4

[30] X. T. Wang, Numerical solution of delay systems containing inverse time by hybrid functions, Appl. Math. Comput., 173 (2006), 535-546. 5.4

[31] S. Q. Wang, J. H. He, Variational iteration method for solving integro-differential equations, Phys. Lett. A, 376 (2007), 188-191. 1

[32] S. P. Yang, A. G. Xiao, Convergence of the variational iteration method for solving multi-delay differential equations, Comput. Math. Appl., 61 (2011), 2148-2151.

[33] E. C. Yen, Solubility and stability of recurrent neural networks with nonlinearity or time-varying delays, Commun. Nonlinear Sci. Numer. Simul., 16 (2011), 509-521. 1

[34] Z. H. Yu, Varaitional iteration method for solving the multi-pantograph delay equation, Phys. Lett. A, 372 (2008), 6475-6479. 